\title{
Determining departure times in dynamic and stochastic maritime routing and scheduling problems
}

\author{
Gregorio Tirado ${ }^{1}$, Lars Magnus Hvattum ${ }^{2}$ \\ ${ }^{1}$ Department of Statistics and Operations Research II (Decision Methods) \\ Universidad Complutense de Madrid, Spain \\ gregoriotd@mat.ucm.es \\ ${ }^{2}$ Faculty of Logistics \\ Molde University College, Norway \\ hvattum@himolde.no
}

\begin{abstract}
In maritime transportation, decisions are made in a dynamic setting where many aspects of the future are uncertain. However, most academic literature on maritime transportation considers static and deterministic routing and scheduling problems. This work addresses a gap in the literature on dynamic and stochastic maritime routing and scheduling problems, by focusing on the scheduling of departure times. Five simple strategies for setting departure times are considered, as well as a more advanced strategy which involves solving a mixed integer mathematical programming problem. The latter strategy is significantly better than the other methods, while adding only a small computational effort.
\end{abstract}

Keywords: tabu search; uncertainty; scenario; maritime transportation.

\section{Introduction}

A dynamic problem is characterized by the fact that new information about the problem arrives while a solution to the problem is being executed (Psaraftis, 1995). In maritime transportation, shipping companies operate a fleet of vessels around the clock, in a never-ending cycle, and new cargoes to transport are revealed to the planners while the vessels follow their planned itineraries. While maritime transportation is a highly dynamic setting, most academic research on optimization based decision support for routing and scheduling of vessels is based on solving static problems (Christiansen et al., 2013). Most research is also considering deterministic optimization problems, more or less neglecting the fact that uncertainty is prevalent in real applications, for example in terms of the appearances of future cargo requests.

This work considers a dynamic and stochastic maritime routing and scheduling problem arising in industrial shipping. The intractability of the problem has led to the development of heuristic solution methods in previous work (Tirado et al., 2013). However, the heuristics used primarily focused on assigning cargoes to vessels and sequencing the cargoes within the itinerary of each vessel. This means that no proper study has been made on the scheduling of departure times in the context of maritime routing and scheduling. That is, the sequence of cargoes dictates to some extent the 
possible departure times, but there may be some flexibility to determine exactly when to departure from a given port.

In a static setting the exact departure times will typically not matter, but in a dynamic problem the choice can influence the ability to deal with future cargoes. This is illustrated in Figure 1 for a case with two known cargoes, C1 and C2, and a single vessel. In the initial plan $\mathrm{C} 1$ is serviced before $\mathrm{C} 2$, and the top left of the figure illustrates an initial plan where departures are made as early as possible, whereas the bottom left illustrates a plan where departures are made as late as possible. If future events are disregarded, the two plans have the same cost. In the middle part, the figure illustrates a potential future situation where a third cargo, C3', becomes known relatively soon and has an early time window. With the early departures (top middle), there is no time to change the planned sequence of visits and C3' cannot be reached on time. However, with late departures (bottom middle), the planner has enough time to re-route the vessel, visiting $\mathrm{C} 3$ ' before $\mathrm{C} 2$, and is therefore able to service all three cargos using the same vessel. Another potential future situation is illustrated to the right, where the third cargo, C3', becomes known relatively late and has a later time window. In this case, the early departures (top right) simply allows cargo C3' to be visited after cargo C2, whereas with late departures (bottom right), the vessel is already too late to service the new cargo.

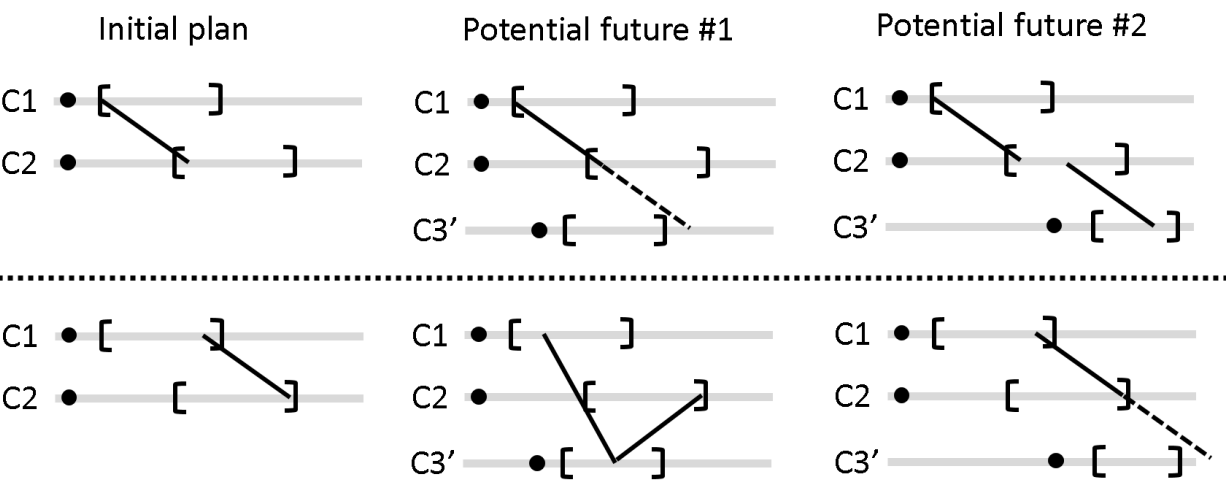

Figure 1: Example showing that the time of departure may influence the ability to service dynamic transportation requests. The brackets indicate time windows for two known and one future cargo operation, the dot indicates the point in time at which information regarding the cargo operations become known, and the black lines indicate travel between ports associated to the cargo operations.

In this paper we investigate the effect of several simple departure time strategies within the context of dynamic and stochastic maritime routing and scheduling. Furthermore, a mixed integer programming model is proposed that can effectively determine departure times taking into account stochastic information about the future, in the form of sampled scenarios. The results show the benefit of the proposed model, as well as the importance of considering departure time scheduling in a dynamic context. 
The remainder of this paper is structured as follows. In Section 2 we define the dynamic and stochastic routing and scheduling problem for which we perform our experiments. Section 3 elaborates on existing literature that considers departure time scheduling for dynamic routing problems. The existing heuristics for making routing decisions in our problem are described in Section 4. Heuristics for making departure time scheduling decisions are then presented in Section 5. A thorough computational study is described and discussed in Section 6, and concluding remarks follow in Section 7.

\section{Problem description}

The dynamic and stochastic maritime pickup and delivery problem considered here was defined in (Tirado et al., 2013). The setting is industrial shipping, where a company operates a fleet of vessels to transport its own cargoes. The vessel fleet is heterogeneous, with vessels varying in terms of speed, cost, and capacity. Each cargo has a given size, and must be transported between a pickup port and a delivery port, with each operation taking place within specified time windows. The company may choose to use an external company to transport any given cargo, through a spot voyage, by paying a lump sum that is a fixed amount per cargo.

The problem is both dynamic and stochastic: additional cargoes will be revealed while the current plan is being executed, but using historical data, probability distributions describing the future cargo requests are available. The objective is therefore not to minimize the costs of transporting the currently known cargoes, but rather to minimize the expected long-term costs of meeting all transportation needs. At any point in time, a plan must be available that specifies how the current cargoes should be transported, either using the company's own fleet or spot voyages. The plan con-

sists of itineraries for each vessel, stating which cargoes to transport, the sequence of pickup and delivery operations, and the time of departures and arrivals. The plan is allowed to change when information arrives about new cargoes, or when a vessel starts a pickup or delivery operation. Once a vessel has started traveling towards a destination, the next destination can no longer be changed. A decision about whether a spot voyage should be used may be changed until the start of the time window of the cargo.

In this work we consider both transportation of full ship loads and parcel loads, the latter involving multi-stop routes. This is relevant for a wide range of settings, such as the transportation of crude oil, iron ore, chemicals, cars, produce and more (Christiansen et al., 2013). The problem is most challenging in the case of multistop routes, as there is more flexibility in terms of assigning different combinations of cargoes to vessels. However, even in the case of full ship loads, decisions regarding departure times and assignments of cargoes to vessels are non-trivial, as illustrated in Figure 1. Taking into account that the vessels of an industrial shipping company operate around the clock throughout the year, there will always be the base that decisions must be made without perfect knowledge about future transportation needs.

To evaluate a policy for making decisions, discrete event simulation is used. The simulator keeps track of the current cargo requests, the positions and history of the 
vessels, and the current plan. Initially, some cargoes are already known, all vessels are empty and located at specific ports ready to start sailing, and an initial plan is created. Whenever a new cargo arrives or a vessel arrives at a port, the simulation clock and the information about cargoes and vessels are updated. At the same time, the plan for servicing known cargoes may be modified. The simulation is run for 360 days, and to compare different solution policies the simulations use common random numbers as a variance reduction technique, so that for a given instance the actual cargo requests arriving are always the same. The main result of the simulation is the total costs calculated as the sum of all sailing costs of all vessels plus the spot voyage costs.

\section{Literature review}

The dynamic and stochastic maritime routing and scheduling problem considered in this paper was defined by Tirado et al. (2013), who tested three different heuristics, namely the myopic dynamic heuristic $(\mathrm{MDH})$, the branch-and-regret heuristic $(\mathrm{BRH})$, and the multiple scenario approach with consensus (MSAC). These heuristics are briefly described in Section 4. The MDH was originally proposed in (Hvattum et al., 2006), the BRH in (Hvattum et al., 2007), and the MSAC in (Bent and Van Hentenryck, 2004). In the implementation of these methods it was assumed that a vessel will depart immediately towards the next destination of its itinerary after performing an operation at a port, and thus the scheduling decisions were given implicitly by the routing decisions.

In other similar problems, waiting has been handled in many different ways in the literature. Ghiani et al. (2009) considered a same-day courier dispatching problem, which is similar to a dynamic and stochastic pickup and delivery problem (PDP), but with the goal of maximizing the customer service level. Samples of requests from the near future are used to evaluate solutions, and waiting time can be inserted into routes as discrete multiples of a given time interval. Mitrović-Minić and Laporte (2004) proposed several simple strategies for a dynamic PDP with time windows (PDPTW), together with a more advanced dynamic waiting strategy based on partitioning the routes in segments containing customers close to each other; the vehicles depart immediately when visiting customers inside the same segment and wait for a certain amount of time when having to move to a different segment. Pureza and Laporte (2008) considered a dynamic PDPTW with random travel times. Several waiting strategies taking advantage of the existence of random travel times were proposed, together with the possibility of buffering requests by delaying their inclusion in the routes. Recently, Vonolfen and Affenzeller (2014) analyzed several waiting policies for the PDPTW, and devised a waiting heuristic that utilizes historical data through an intensity measure.

Bent and Hentenryck (2007) developed a waiting and relocation strategy for a dynamic and stochastic vehicle routing problem with time windows (VRPTW) based on the solutions of a set of scenarios: if the next customer to be visited is a sampled customer in many of the scenarios, the vehicle waits. Otherwise, it departs towards the next location. Saint-Guillain et al. (2015), considered another dynamic and stochastic 
VRPTW where assignment and routing decisions were based on a multistage stochastic programming formulation. In this context the authors evaluated some simple strategies for scheduling departures, and found that it was advisable to depart immediately after visiting a customer node, while waiting as long as possible after a relocation move performed to locate the vehicle in a more convenient location. Ghiani et al. (2008) applied drive-first and wait-first policies to a dynamic and stochastic traveling salesman problem (TSP) in which the objective is to maximize the service level and not the traveled distance.

Branke et al. (2005) developed an evolutionary algorithm to search over the space of all possible waiting strategies, for a vehicle routing problem (VRP) where a single new customer arrives after the vehicles have left the depot. Ichoua et al. (2006) considered a real-time vehicle dispatching problem, with soft time windows and no vehicle capacities. The vehicles wait a certain amount of time that is proportional to the probability of having new requests in the neighborhood of the current location. This time is bounded to ensure that the vehicle is able to arrive on time back to the depot. Thomas (2007) studied waiting strategies for anticipating service requests from known customer locations, focusing on where to wait to facilitate late request customers. The objective is to maximize the number of customers that can be served, and the problem is considered for a single vehicle with no capacity restriction. Branchini et al. (2009) proposed a granular local search heuristic for a dynamic VRP, using a wait-first strategy at each location and returning to a previously defined strategic waiting point when no customer visits are scheduled. Ferrucci et al. (2013) approached a dynamic VRP with soft time windows, dealing with the delivery of urgent goods, in which the available information is used to guide vehicles to request-likely areas where the vehicles wait for new requests to arrive, instead of just waiting at the last customer location.

There are many additional publications addressing dynamic or stochastic variants of routing and scheduling problems. Three recent surveys on dynamic vehicle routing problems were presented by Pillac et al. (2013), Bektas et al. (2014) and Psaraftis et al. (2015). A review of stochastic vehicle routing problems was written by Berhan et al. (2014), and finally, a survey of dynamic and stochastic vehicle routing problems was presented by Ritzinger et al. (2015).

\section{Heuristics for routing decisions}

This section will describe briefly the existing solution methods for routing decisions introduced in Tirado et al. (2013) to solve the problem approached in this paper. The methods do not explicitly take into account decisions regarding scheduling of the departures of the vessels, instead assuming that they depart immediately after finishing a pickup or delivery operation. Under this assumption, a new plan is created every time a vessel arrives at a port or some new cargo request is received.

The myopic dynamic heuristic (MDH) applies a tabu search algorithm (Korsvik et al., 2010) at each decision point to solve a static and deterministic optimization problem containing only the cargoes that are known with certainty at that moment. Hence, it does not use any stochastic information regarding possible future cargo re- 
quests. Tirado et al. (2013) proposed a mathematical programming model for this static and deterministic pickup and delivery problem which is solved at each MDH iteration heuristically. The tabu search used to solve these subproblems uses a neighborhood based on the relocation of cargoes from one vessel to another.

The multiple scenario approach with consensus (MSAC) is a solution method that makes use of the stochastic information available regarding future cargo requests in the form of scenarios. At each decision point a new set of scenarios is generated. They always contain all cargoes known with certainty, in addition to some sampled cargoes generated according to the associated probability distributions. The scenarios represent different possible outcomes of the random variables of the problem and are used to anticipate likely future cargo requests. Each scenario is then solved using the same tabu search algorithm as in MDH, and one of those scenario solutions is chosen according to a consensus function. This function measures how similar a scenario solution is with respect to the other scenario solutions when considering only the first cargo serviced by each vessel. As a result, the scenario solution which is most similar to the others is selected, and after dropping its future sampled cargoes, it becomes the new plan for the currently known cargoes.

The branch-and-regret heuristic (BRH) also uses stochastic information in the form of scenarios. However, the new plan is not based on one single scenario solution, but is iteratively built by resolving the scenarios several times by adding additional constraints so that the scenario solutions finally converge to one single solution regarding how the known cargoes are serviced. In the first phase of the algorithm, the constraints added to the scenarios concern whether a given cargo must be serviced by a given vessel or by any other. As a result, when this first phase is completed, all known cargoes are serviced by the same vessel in all scenario solutions, even though the sequence followed by the vessels may differ. In the second phase of the algorithm, constraints related to the sequence followed by each vessel to service the known cargoes are iteratively added, ending up with a set of scenario solutions with identical sequences regarding the known cargoes. These sequences provide the new plan for the currently known cargoes once all sampled cargoes are dropped. The subproblems solved at each iteration of BRH are also solved using the same tabu search algorithm as in MDH and MSAC, with the appropriate modifications to take into account the additional constraints added in each case.

\section{$5 \quad$ Heuristics for scheduling decisions}

The strategy used by default in our previous work (Tirado et al., 2013), which will be referred to as Depart Immediately (DI), consists of departing immediately from the current position and move towards the next planned port call. We assume that once a vessel starts moving towards a port to perform a pickup or delivery operation, it cannot be diverted until the planned service is finished. If the vessel arrives inside the time window, service starts immediately, but if it arrives before the beginning of the time window, it must wait at the destination without being available to be diverted to serve other cargoes. This strategy was intended to maximize the utilization of the fleet, but could result in unnecessary waiting times at the destinations, where the 
vessels cannot be diverted. To facilitate the insertion of new cargo requests into the current plan other alternative waiting strategies are considered, based on deciding when it is convenient for a vessel to wait at the origin port before sailing towards the destination in order to be diverted in the future if an appropriate cargo request arrives.

\subsection{Simple waiting strategies}

The first alternative strategy considered, called Earliest Arrival (EA), consists of waiting at the current position until a time such that the arrival at the next planned port call happens as early as possible but not earlier than the beginning of the associated time window. When departing immediately leads to an arrival that happens within the time window associated to the destination, DI and EA coincide. Otherwise, the waiting time that is spent at the destination when using DI, can be spent at the origin by using EA, thereby leading to the advantage of being able to divert the vessel during that time if it is beneficial.

Another possibility, referred to as Latest Arrival (LA), is to wait at the current position as long as possible without missing the end of any time windows for a currently planned port call. In this way the services planned are delayed as much as possible to wait for appropriate new cargo requests.

The convenience of following EA or LA is very much related to the current sailing plan, in particular with respect to the load of the vessels upon departure and the distance to be traveled. For example, if a vessel is heavily loaded after performing service at the current port, it is very unlikely that it could be diverted to serve any new cargo request due to capacity restrictions, because some delivery operations should be performed first, and thus waiting at the current port would not be beneficial. Based on this idea we define the Load Based waiting strategy (LB), according to which EA would be followed if the vessel is currently loaded up to more than $\alpha \%$ of the total capacity, and LA would be followed otherwise. In this work, the threshold has been set according to $\alpha=50$.

Another idea, already used in the literature before (Mitrović-Minić and Laporte, 2004), is to make the decision based on the distance to the next location: if it is close, the potential saving that could be achieved by waiting would be small, making it less interesting, while if it is far away larger savings could be achieved if an appropriate cargo request is received while waiting. Based on this we developed the Distance Based waiting strategy (DB), which consists of following EA if the next port is closer to the current position than the average distance between ports, and follow LA otherwise.

Figure 2 illustrates waiting strategies DI (dashed line), EA (solid line), and LA (dotted line). The planned route starts in port 1, then visits in sequence ports 2,4 , 3 , and 5 before ending by going back to port 4 . Time windows for the operations are represented by brackets.

Apart from the simple strategies presented above, we have also developed another more sophisticated strategy, called Model Based (MB) strategy, based on solving a mathematical programming model to determine suitable departure times for the vessels. In this model the decisions regarding routing have been fixed, but there is some flexibility in the decisions regarding departure times. A set of possible future 


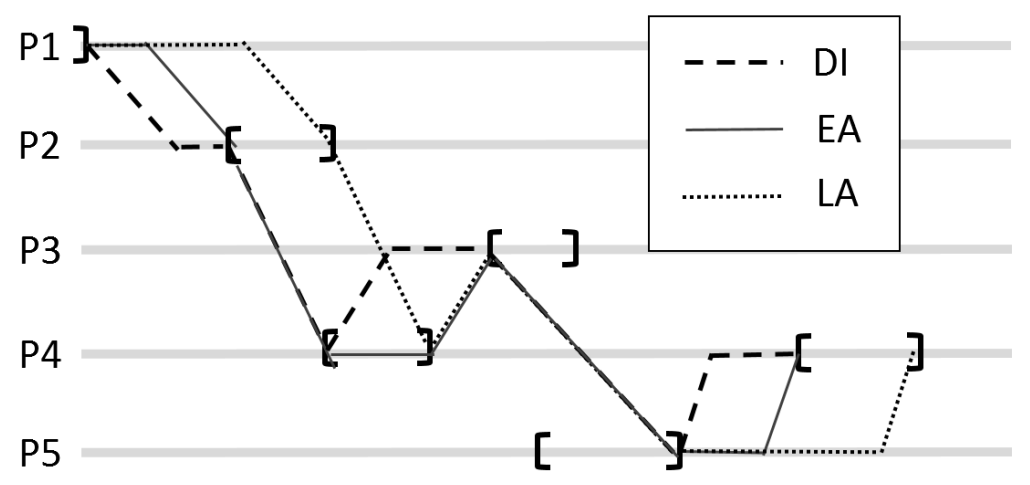

Figure 2: Illustration of simple waiting strategies

cargoes is considered, together with a subset of already known cargoes, and the model finds departure times so as to maximize the benefit of being able to either service additional cargoes or to change how current cargoes are serviced. This could be seen as a two-stage problem, where the first stage decisions are the departure times from the current positions, and where the second stage decisions are related to how future cargoes are inserted into the routes while making sure that the related scheduling decisions are feasible. The mathematical model of the MB strategy is detailed in the following section.

\subsection{Mathematical model based waiting strategy}

At a certain point in time, a set $R$ of planned routes is given, with one route for each vessel. Each route $r \in R$ consists of a number of planned port visits, $i \in N_{r}=$ $\left\{1, \ldots,\left|N_{r}\right|\right\}$. Let the travel time between visits $i$ and $i+1$ of route $r$ be denoted by $T_{r i}$, with $T_{r\left|N_{r}\right|}=0$. There is a time window for each port visit, such that the arrival time $t_{r i}$ associated to port visit $i$ on route $r$ must be within $\left[A_{r i}, B_{r i}\right]$.

The goal is to find arrival times, $t_{r i}$, that maximize the potential to service future cargo requests and the flexibility to change the current set of routes. A set $S$ of potential cargo requests is given, for example by sampling from the probability distributions describing future cargo requests. Each sampled cargo request $s$ has a time window for the pickup operation $\left[A_{s}^{P}, B_{s}^{P}\right]$, and a time window for the delivery operation $\left[A_{s}^{D}, B_{s}^{D}\right]$. Let the travel time between the pickup port of $s$ and the port corresponding to visit $i$ on route $r$ be denoted by $T_{r s i}^{P}$, and similarly let $T_{r s i}^{D}$ be the travel time between the delivery port of $s$ and visit $i$ on route $r$. Furthermore, let $T_{r s}^{P D}$ be the travel time between the pickup port and the delivery port of cargo request $s$ using the vessel of route $r$. If the model decides to schedule a route $r$ such that cargo request $s$ could be serviced, $t_{r s}^{P}$ and $t_{r s}^{D}$ will correspond to arrival times for the pickup and delivery ports, respectively.

Let $x_{r i j s}$ be a binary variable indicating whether cargo $s$ could be serviced by route $r$ with the pickup operation immediately after visit $i$ and the delivery operation after 
visit $j$. If $i=j$, the delivery operation of cargo $s$ could be performed immediately after the pickup operation. Let $F_{r s} \subseteq N_{r} \times N_{r}$ be the set of pairs $(i, j)$ such that it is feasible with respect to capacity, compatibility, and precedence constraints to service cargo $s$ on route $r$ as described by $x_{\text {rijs }}$. Let $U_{\text {rijs }}$ be a measure of the benefit of being able to insert cargo $s$ as indicated by $x_{r i j s}$, and let $G_{r s}=\left\{(i, j) \in F_{r s}: U_{r i j s}>0\right\}$. Let $C_{r i}^{T}$ be the travel cost between visit $i$ and visit $i+1$ on route $r, C_{r i s}^{P}$ the travel cost between visit $i$ and the pickup operation of cargo $s, C_{\text {ris }}^{D}$ the travel cost between visit $i$ and the delivery operation of cargo $s, C_{r s}^{P D}$ the travel cost between the pickupoperation and the delivery operation of cargo $s$, and let $C_{s}$ be the cost of being unable to service cargo $s$. The travel costs depend on the vessel assigned to the route, whereas the cost of being unable to service a cargo is equal to the cost of using a spot voyage instead of using the company's own fleet. When $i \neq j$ the benefit can be written as $U_{\text {rijs }}=C_{s}-C_{r i s}^{P}-C_{r(i+1) s}^{P}+C_{r i}^{T}-C_{r j s}^{D}-C_{r(j+1) s}^{D}+C_{r j}^{T}$, and with $i=j$ the benefit can be expressed as $U_{\text {riis }}=C_{s}-C_{r i s}^{P}-C_{r s}^{P D}-C_{r(i+1) s}^{D}+C_{r i}^{T}$.

The important outputs from the model are the departure times from the current locations, calculated as $t_{r, 2}-T_{r, 1}$. That is, the model specifies when one should arrive the next destination, $t_{r, 2}$, and from this one can calculate when it is necessary to depart from the current location. The other departure times are important to ensure feasibility, but may change when re-optimizing the plans at a later point in time. If the model is unable to facilitate service of additional cargoes on a given route, the quality of different arrival times cannot be discerned. That is, there are multiple optimal solutions where the value of $t_{r, 2}$ differs. To support defaulting to either of EA, LA, LB, or DB, a small coefficient $m_{r}$ is added to the objective function for each of the arrival times $t_{r, 2}$. For example, setting $m_{r}=-\frac{U_{r}^{M I N}}{\left(B_{r, 2}-A_{r, 2}\right)}$, where $U_{r}^{M I N}=\min _{s \in S} \min _{(i, j) \in G_{r s}} U_{r i j s}$, will make sure that EA is followed when no additional cargoes can be planned for route $r$.

$\max \sum_{r \in R} \sum_{s \in S} \sum_{(i, j) \in G_{r s}} U_{r i j s} x_{r i j s}+\sum_{r \in R:\left|N_{r}\right|>1} m_{r} t_{r, 2}$ 
s.t

$\sum_{r \in R} \sum_{(i, j) \in G_{r s}} x_{r i j s} \leq 1, s \in S$,

$\sum_{s \in S} \sum_{(i, j) \in G_{r s}} x_{r i j s} \leq 1, r \in R$,

$A_{r i} \leq t_{r i} \leq B_{r i}, r \in R, i \in N_{r}$,

$A_{s}^{P} \leq t_{r s}^{P} \leq B_{s}^{P}, r \in R, s \in S$,

$A_{s}^{D} \leq t_{r s}^{D} \leq B_{s}^{D}, r \in R, s \in S$,

$t_{r(i+1)} \geq t_{r i}+T_{r i}, r \in R, i \in N_{r} \backslash\left\{\left|N_{r}\right|\right\}$,

$t_{r s}^{P} \geq t_{r i}+T_{r i s}^{P}-\left(B_{r i}+T_{r i s}^{P}\right)\left(1-x_{r i j s}\right), r \in R, s \in S,(i, j) \in G_{r s}$,

$t_{r(i+1)} \geq t_{r s}^{P}+T_{r(i+1) s}^{P}-\left(B_{s}^{P}+T_{r(i+1) s}^{P}\right)\left(1-x_{r i j s}\right), r \in R, s \in S,(i, j) \in G_{r s}: j \neq i \neq\left|N_{r}\right|$,

$t_{r s}^{D} \geq t_{r j}+T_{r j s}^{D}-\left(B_{r j}+T_{r j s}^{D}\right)\left(1-x_{r i j s}\right), r \in R, s \in S,(i, j) \in G_{r s}: i \neq j$,

$t_{r s}^{D} \geq t_{r s}^{P}+T_{r s}^{P D}-\left(B_{s}^{P}+T_{r s}^{P D}\right)\left(1-x_{r i i s}\right), r \in R, s \in S,(i, i) \in G_{r s}$,

$t_{r(j+1)} \geq t_{r s}^{D}+T_{r(j+1) s}^{D}-\left(B_{s}^{D}+T_{r(j+1) s}^{D}\right)\left(1-x_{r i j s}\right), r \in R, s \in S,(i, j) \in G_{r s}: j \neq\left|N_{r}\right|$,

$x_{\text {rijs }} \in\{0,1\}, r \in R, s \in S,(i, j) \in G_{r s}$.

The objective function (1) adds up the benefits from being able to insert additional cargoes in the planned routes. It additionally includes terms that induce a default waiting strategy whenever the model is unable to insert any additional cargoes. Constraints (2) state that each additional cargo is limited to be serviced once, while constraints (3) state that each route should only accommodate one additional cargo.

Constraints (4)-(6) make sure that the arrival times of planned visits, additional pickup operations, and additional delivery operations are within the respective time windows. The following constraints make sure that travel times between consecutive visits are included when calculating arrival times. Constraints (7) restrict the arrival time at visit $i+1$ of route $r$, based on the arrival time at visit $i$. The arrival time for the pickup operation of cargo $s$ on route $r$, if the pickup is planned after regular visit $i$, is restricted by constraints (8). When the pickup operation of cargo $s$ on route $r$ is planned immediately before visit $i+1$, constraints (9) limit the arrival time at visit $i+1$. Constraints (10) handle the arrival time of the delivery operation of cargo $s$ when planned immediately after visit $j$ on route $r$, and constraints (11) handle the arrival time of the delivery operation when planned immediately after the pickup operation. The arrival time at visit $j+1$ on route $r$ when following a delivery operation of cargo $s$ is restricted by constraints (12). Finally, constraints (13) specify the integrality restriction on the $x$-variables.

Some variations of the model (1)-(13) may be worth considering. By dropping constraints (2), each cargo may be covered by several routes. This is advantageous in case several similar cargoes appear in the near future. On the other hand, by 
dropping constraints (3) one route may accommodate several possible future cargoes, which is advantageous in terms of increasing flexibility. By dropping both constraints (2) and constraints (3), both advantages may be realized. However, in these cases, the interpretation of the coefficients in the objective function may change. Furthermore, when dropping constraints (2) the problem decomposes into independent subproblems for each route.

Computational experiments are needed to evaluate, i) the four different model variations depending on how constraints (2)-(3) are treated, ii) which default waiting strategy to use when the model is unable to accommodate any additional cargoes on a route, iii) how to compose the set $S$ of cargoes, including the size of the set as well as whether to include only additionally sampled cargoes or also existing cargoes from different routes, and iv) to find appropriate settings for the MIP solver used to solve the model, in terms of time limits and acceptable optimality gaps.

\section{Computational study}

This section presents the computational study performed to evaluate and analyze the performance of the proposed waiting strategies. The simulator and the associated solution methods were implemented in $\mathrm{C}++$ and the MB strategy mathematical model was solved using Xpress using the Builder Component Library. Computations were carried out using a cluster with nodes each consisting of twelve $2.4 \mathrm{GHz} 64$ bit CPUs from AMD, and with 24 GB RAM per node, running under Linux.

The rest of this section is organized as follows. First, Section 6.1 describes the test instances used in the computational study. Section 6.2 compares the results obtained with the different simple strategies proposed in the paper. Section 6.3 summarizes the calibration experiments performed to find appropriate parameter settings for the mathematical model associated to strategy MB. Finally, Section 6.4 presents a compilation of the results obtained for each instance group with both the best simple strategy and the MB strategy.

\subsection{Test instances}

It is desirable to test the waiting strategies in the context of real instances collected from industrial shipping companies (Silberholz and Golden, 2010). However, since it would be prohibitively time consuming to collect this data for a sufficiently large number of cases, we resort to generating random test instances with realistic features. The instances are based on real distances between ports, and real ship data, as in (Hemmati et al., 2014). Each port is designated either as a production port, where goods must be picked up, or as a consumption port where goods are to be delivered, and $25 \%$ of the ports are selected as production ports. Each pair of production and consumption ports has a given probability (possibly zero) of generating a new cargo request per day, but there is also a minimum number of days between consecutive cargo requests between two given ports. Time windows are generated for each cargo request based on the distance between ports. Uniform probability distributions are used for the cargo size and the notification time, that is the number of days prior 
to the pickup time window that the request will be known. The vehicle fleet may consist of up to three different types of ships, where the capacity of the largest ship is slightly less than twice that of the smallest ship. For partial load instances, the size of each cargo is random but scaled so that the largest ship can carry four average sized cargoes simultaneously.

As the value of different waiting strategies may depend on the structure of the test instances used, two different sets of instances were considered. The first set, called Set1, corresponds to the set with medium spot voyage costs from (Tirado et al., 2013). These instances vary based on the fleet size (small size fleet with 6 vessels, medium size fleet with 12 vessels, or large size fleet with 24 vessels), the fleet composition (heterogeneous, with vessels with different costs and capacities, or homogeneous with only one type of vessel), the size of cargoes (full load or partial load), the number of cargoes (high or low demand), and the number of ports (either 16 or 32 ports). For each combination of attributes, two distinct instances were generated, resulting in a total of 96 different instances.

The second set, called Set2, was generated in the same way but with instances where the size of the time windows and the notification times associated to the cargo requests are varied, so that their effect on the importance of the scheduling decisions can be estimated. Set 2 contains a total of 72 instances with varying characteristics: parcel or full load; big, medium, or small size; high or low demand; narrow or wide time windows (10 to 20 or 15 to 30 days for the pickup time window); and short, medium, or long notification times ( 5 to 20,15 to 40 , or 25 to 60 days). In addition, a small set of instances for the calibration was also generated, independently of Set1 and Set2.

\subsection{Results of simple strategies}

This section presents a comparison of the results obtained with the different simple waiting strategies proposed in this paper (EA, LA, LB, and DB) with respect to the default strategy (DI) used in previous work (Tirado et al., 2013). Table 1 displays the savings provided by each strategy with respect to the default one for each solution method (MDH, MSAC, BRH). Positive numbers corresponds to cost savings, and negative numbers represent an increase in total costs. The instances of both Set 1 and Set2 are aggregated in parcel load and full load instances, and average percentage cost savings are given for each group.

\begin{tabular}{rr|rrrr} 
& & EA & LA & LB & DB \\
\hline MDH & parcel & $0.406 \%$ & $-3.894 \%$ & $-0.283 \%$ & $-0.549 \%$ \\
& full load & $0.423 \%$ & $-1.626 \%$ & $-0.078 \%$ & $-1.162 \%$ \\
\hline MSAC & parcel & $0.400 \%$ & $-6.395 \%$ & $-1.353 \%$ & $-0.226 \%$ \\
& full load & $0.621 \%$ & $-1.458 \%$ & $-0.026 \%$ & $-0.663 \%$ \\
\hline \multirow{2}{*}{ BRH } & parcel & $1.071 \%$ & $-3.422 \%$ & $-0.199 \%$ & $0.125 \%$ \\
& full load & $0.317 \%$ & $-1.473 \%$ & $-0.332 \%$ & $-0.952 \%$
\end{tabular}

Table 1: Average percentage cost savings provided by the proposed waiting strategies with respect to DI. 
Table 1 shows that EA clearly outperforms DI, with the improvement being highest on parcel load instances when using BRH. However, the other strategies perform worse than DI in average, even though they provide positive savings for some particular instances. There is a significant cost increase when using LA, indicating that always delaying departures as much as possible is definitely not a good strategy in this setting.

These results allow us to conclude that the only simple strategy improving over the default is EA, highlighting the difficulty of making good scheduling decisions in this problem and suggesting that more sophisticated waiting strategies would be needed. As a result, we chose EA as the default waiting strategy in MB, to use when the mathematical model is unable to accommodate any additional cargoes on a route, and we will focus the rest of the computational study on comparing the performance of the best simple strategy, EA, with the one based on the mathematical model, MB.

\subsection{Calibration}

The calibration study to find a good set of parameters for the MB strategy has been performed on a reduced set of seven instances, similar to the one used in (Tirado et al., 2013), generated independently of the instances used to evaluate the performance of the waiting strategies.

First, we solved the calibration instances using the four different model variations depending on how constraints (2)-(3) are treated. If only one of those constraint sets is removed, the running time required to solve the model is approximately the same as for the complete model, but the results are worse overall. Furthermore, when both sets of constraints are relaxed simultaneously, the running time required by the solver increases significantly, probably due to the high increase in the number of feasible solutions, but again the final solutions do not become better. This indicates that the best overall performance is obtained when both sets of constraints are included in the model, and thus the complete model is used in all remaining experiments.

Second, we focused on how to compose the set $S$ of cargoes of the model: the number of different scenarios to generate, the number of sampled cargoes per scenario, and the total amount of existing cargoes from different routes to be considered. We first solved all calibration instances when varying the number of scenarios in $\{10,20, \ldots, 50\}$ and the number of sampled cargoes per scenario in $\{0,2, \ldots, 14\}$, while fixing the number of existing cargoes to be added to five. The average percentage saving obtained with each combination is shown in Figure 3, from which we conclude that twenty scenarios and around four sampled cargoes per scenario seem enough, while increasing them further does not provide significant additional improvements. As a result, we decided to fix the number of scenarios to twenty and then vary the number of existing cargoes in $\{0,2, \ldots, 12\}$ and the number of sampled cargoes in $\{0,4, \ldots, 20\}$. The results, shown in Figure 4 , allowed us to confirm that four sampled cargoes per scenario is enough and that it is not worthwhile to increase it further, and that using more than four existing cargoes does not help. Hence, in what follows the model of the MB strategy will always be built using twenty scenarios, with four sampled cargoes per scenario and four existing cargoes.

Third, the settings of the MIP-solver used to solve the model may also influence the results. Throughout the tests reported here, the solver is allowed to use only one 


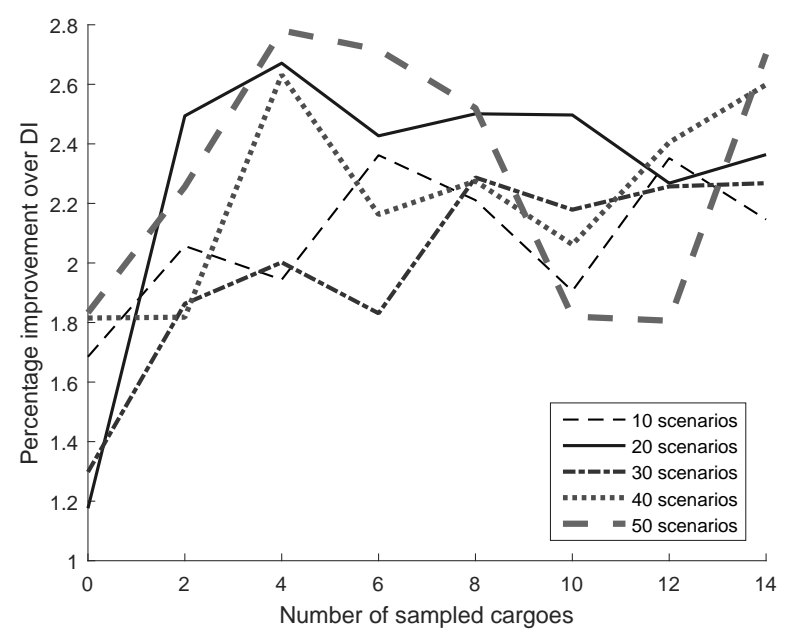

Figure 3: Results from calibration tests with a fixed number of five existing cargoes added from each existing route, and with varying number of sampled cargoes included.

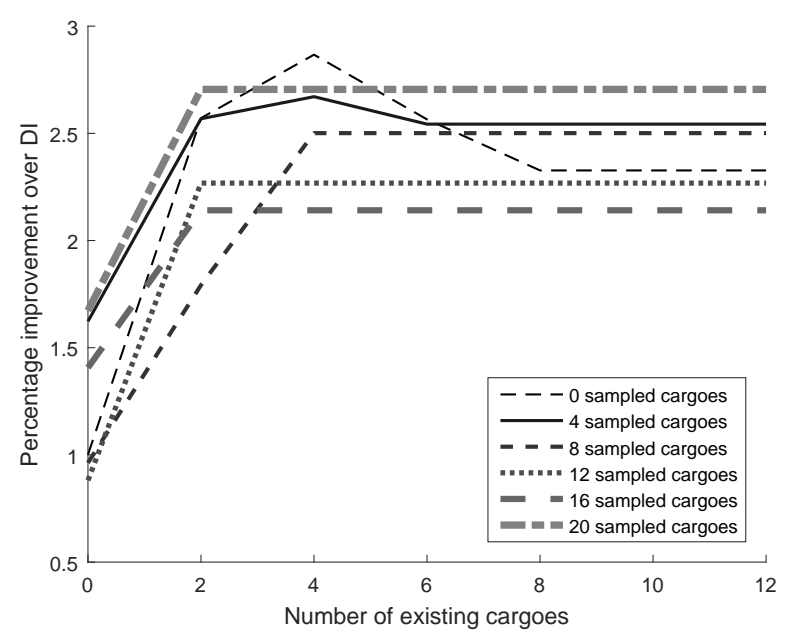

Figure 4: Results from calibration tests varying the number of existing and sampled cargoes included in the model, with twenty scenarios from which sampled cargoes are generated.

thread, it terminates when the relative gap between the upper and lower bound is less than 0.001, and there is a maximum time limit of thirty seconds. If no feasible solution is found after thirty seconds, the departure time decisions are made based on the default strategy instead. For other parameters, the default settings are kept. 


\subsection{Main results}

This section contains an analysis of the performance of the MB strategy in comparison with EA, to evaluate the potential benefits of using a mathematical model to make scheduling decisions. For this purpose, average improvements of MB over EA are given for several subsets of instances, with the aim of analyzing the influence of different instance attributes on the relative performance of MB. Both Set1 and Set2 are partitioned into parcel or full load instances; big, medium, or small sized instances; and high or low demand instances. Additionally, Set1 is also partitioned into instances with 16 or 32 ports, while Set2 is also partitioned into narrow or wide time windows (TWs) and large, medium, or small notification times (NTs).

The results for parcel and full load instances of Set1 are presented in Tables 2 and 3 , respectively. Similarly, the results for parcel and full load instances of Set 2 are given in Tables 4 and 5, respectively. The first two columns indicate the instance subset considered in each case and the number of instances in that subset, respectively, and each column pair corresponds to one solution method among MDH, MSAC, and BRH. The first column of each pair shows the average percentage cost saving achieved by $\mathrm{MB}$ with respect to $\mathrm{EA}$, where positive values indicate that $\mathrm{MB}$ performed better than EA. The second column of each pair shows the P-Value of a one sided t-test regarding whether significant cost savings are achieved when using MB waiting strategy to make scheduling decisions instead of EA.

The results regarding MDH are omitted in Tables 3 and 5. This is done because the final solutions obtained for all full load instances when using MDH together with EA or MB waiting strategies were identical. This indicates that, when MDH solution method is used, the scheduling decisions made by the mathematical model on full load instances match the default strategy EA at each decision point and thus no further savings are achieved. This could be due to the fact that MDH does not consider stochastic information and thus it is more difficult for the plans designed at each decision point to serve new cargo requests by delaying the departure of vessels. Besides, in full load instances the load of several cargo requests cannot be loaded into the vessel simultaneously, making it even more difficult to include new cargo requests into the plans.

Tables $2-5$ show that the largest savings are obtained when using the methods that consider stochastic information, MSAC and $\mathrm{BRH}$, on big and low demand instances. This happens because MSAC and BRH usually provide plans with a higher flexibility than MDH to serve future cargo requests by changing the scheduling decisions, allowing MB strategy to provide larger additional savings. Similarly, making better scheduling decisions is, in general, more important on bigger instances. Furthermore, if the demand is high, the vessels of the fleet are sailing most of the time and delaying departures usually does not pay off. As a result, low demand instances have a higher potential for additional savings when using MB strategy to make scheduling decisions.

In general we observe that the number of ports or the size of the time windows do not seem to have a large effect on the potential savings provided by the MB strategy. However, the notification time does, with smaller notification times leading to larger savings provided by MB. A possible explanation is that when the notification time is large enough, there is plenty of time to replan and delaying departures is not necessary. 
However, if the notification times are small, anticipating the arrival of cargo requests by performing the appropriate scheduling decisions can provide significant savings.

\begin{tabular}{r|r|rr|rr|rr} 
Subset & \# inst & MDH & P-Value & MSAC & P-Value & BRH & P-Value \\
\hline Big & 16 & $0.52 \%$ & 0.083 & $1.98 \%$ & 0.006 & $1.32 \%$ & 0.000 \\
Medium & 16 & $0.60 \%$ & 0.054 & $0.75 \%$ & 0.090 & $1.10 \%$ & 0.022 \\
Small & 16 & $0.90 \%$ & 0.085 & $0.08 \%$ & 0.451 & $0.39 \%$ & 0.337 \\
High demand & 24 & $0.66 \%$ & 0.059 & $-0.18 \%$ & 0.329 & $0.17 \%$ & 0.368 \\
Low demand & 24 & $0.68 \%$ & 0.027 & $2.05 \%$ & 0.000 & $1.70 \%$ & 0.001 \\
16 ports & 24 & $1.58 \%$ & 0.000 & $0.68 \%$ & 0.126 & $1.40 \%$ & 0.005 \\
32 ports & 24 & $-0.24 \%$ & 0.178 & $1.19 \%$ & 0.007 & $0.47 \%$ & 0.184 \\
\hline All & 48 & $0.67 \%$ & 0.007 & $0.94 \%$ & 0.007 & $0.93 \%$ & 0.006
\end{tabular}

Table 2: Comparing results of EA and MB for the original parcel load instances, reporting the percentage improvement of results with MB compared with EA.

\begin{tabular}{r|r|rr|rr} 
Subset & \# inst & MSAC & P-Value & BRH & P-Value \\
\hline Big & 16 & $-0.07 \%$ & 0.204 & $-0.04 \%$ & 0.246 \\
Medium & 16 & $0.27 \%$ & 0.178 & $0.01 \%$ & 0.460 \\
Small & 16 & $-0.42 \%$ & 0.193 & $0.26 \%$ & 0.088 \\
High demand & 24 & $0.04 \%$ & 0.337 & $0.14 \%$ & 0.041 \\
Low demand & 24 & $-0.18 \%$ & 0.305 & $0.01 \%$ & 0.465 \\
16 ports & 24 & $-0.14 \%$ & 0.346 & $0.12 \%$ & 0.156 \\
32 ports & 24 & $0.00 \%$ & 0.493 & $0.02 \%$ & 0.382 \\
\hline All & 48 & $-0.07 \%$ & 0.351 & $0.07 \%$ & 0.154
\end{tabular}

Table 3: Comparing results of EA and MB for the original full load instances, reporting the percentage improvement of results with MB compared with EA. For the MDH method, using EA and MB yields identical results.

\section{Concluding remarks}

Dynamic and stochastic routing and scheduling problems have received relatively little attention in the academic literature, compared to their static and deterministic counterparts. In maritime transportation, real life applications are typically associated to high degrees of uncertainty while decisions must be made with incomplete knowledge of future transportation tasks. Previous research has established that taking into account information about uncertain future cargoes will lead to consistent and large cost savings, compared to an approach where static and deterministic problems are solved repeatedly in response to new information.

This work addressed a gap in the previous literature on dynamic and stochastic maritime routing problems, by investigating the role of scheduling departure times for vessels. Five simple strategies for assigning departure times have been tested: 1) departure immediately as soon as the next port visit is determined, 2) departure 


\begin{tabular}{r|r|rr|rr|rr} 
Subset & \# inst & MDH & P-Value & MSAC & P-Value & BRH & P-Value \\
\hline Big & 12 & $-0.31 \%$ & 0.312 & $2.09 \%$ & 0.019 & $1.50 \%$ & 0.067 \\
Medium & 12 & $0.18 \%$ & 0.436 & $2.65 \%$ & 0.006 & $2.47 \%$ & 0.020 \\
Small & 12 & $1.61 \%$ & 0.189 & $1.96 \%$ & 0.048 & $1.62 \%$ & 0.114 \\
High demand & 18 & $0.41 \%$ & 0.346 & $1.53 \%$ & 0.044 & $0.44 \%$ & 0.281 \\
Low demand & 18 & $0.57 \%$ & 0.289 & $2.93 \%$ & 0.000 & $3.29 \%$ & 0.001 \\
Narrow TW & 18 & $-0.24 \%$ & 0.422 & $1.64 \%$ & 0.021 & $2.32 \%$ & 0.009 \\
Wide TW & 18 & $1.23 \%$ & 0.066 & $2.83 \%$ & 0.001 & $1.41 \%$ & 0.062 \\
Small NT & 12 & $0.45 \%$ & 0.414 & $4.03 \%$ & 0.003 & $4.13 \%$ & 0.006 \\
Medium NT & 12 & $0.76 \%$ & 0.200 & $1.78 \%$ & 0.020 & $1.39 \%$ & 0.034 \\
Large NT & 12 & $0.27 \%$ & 0.132 & $0.89 \%$ & 0.038 & $0.08 \%$ & 0.459 \\
\hline All & 36 & $0.49 \%$ & 0.246 & $2.23 \%$ & 0.000 & $1.86 \%$ & 0.002
\end{tabular}

Table 4: Comparing results of EA and MB for the new parcel load instances

\begin{tabular}{r|r|rr|rr|} 
Subset & \# inst & MSAC & P-Value & BRH & P-Value \\
\hline Big & 12 & $0.13 \%$ & 0.268 & $-0.18 \%$ & 0.183 \\
Medium & 12 & $-0.37 \%$ & 0.015 & $0.70 \%$ & 0.124 \\
Small & 12 & $-0.90 \%$ & 0.105 & $-0.36 \%$ & 0.185 \\
High demand & 18 & $-0.18 \%$ & 0.191 & $0.20 \%$ & 0.144 \\
Low demand & 18 & $-0.58 \%$ & 0.105 & $-0.10 \%$ & 0.418 \\
Narrow TW & 18 & $-0.69 \%$ & 0.073 & $0.12 \%$ & 0.405 \\
Wide TW & 18 & $-0.07 \%$ & 0.345 & $-0.01 \%$ & 0.456 \\
Small NT & 12 & $-0.06 \%$ & 0.403 & $0.68 \%$ & 0.135 \\
Medium NT & 12 & $-0.64 \%$ & 0.149 & $-0.04 \%$ & 0.403 \\
Large NT & 12 & $-0.44 \%$ & 0.140 & $-0.48 \%$ & 0.108 \\
\hline All & 36 & $-0.38 \%$ & 0.064 & $0.05 \%$ & 0.415
\end{tabular}

Table 5: Comparing results of EA and MB for the new full load instances 
as early as possible without arriving earlier than the beginning of the time window of the next request (EA), 3) departure as late as possible without missing any time windows (LA), 4) select a mix of EA and LA based on the current load of the vessel, and 5) select a mix of EA and LA based on the distance to the next planned port visit. Among these, the EA strategy was shown to be clearly superior.

Furthermore, a mathematical model was developed to make departure time decisions. The model includes information about future cargo requests in the form of sampled scenarios. Computational experiments reveal that a strategy for assigning departure times based on the model is superior to the best simple strategy. It is also observed that the importance of using the model is higher in situations with short notification times, as well as in situations with low demand. The model can be used as a post processing of solutions where routing and assignment decisions have been fixed. However, the effect of using the model (as opposed to EA) is greater when the routing decisions have been made by heuristics incorporating stochastic information. Presumably this holds because those routing decisions to a larger degree supports flexibility in handling future events, which the mathematical model for making scheduling decisions can exploit.

Acknowledgements This research was carried out with financial support from the DOMinant II project, partly funded by the Research Council of Norway, from a grant from Iceland, Liechtenstein and Norway through the EEA Financial Mechanism (operated by Universidad Complutense de Madrid) with reference 026-ABELIM-2013, and from the Government of Spain, grant TIN2012-32482.

\section{References}

T. Bektas, P. P Repoussis, and C.D. Tarantilis. Dynamic vehicle routing problems. In P. Toth and D. Vigo, editors, Vehicle Routing: Problems, Methods, and Applications, MOS-SIAM Series on Optimization, pages 299-348. Society for Industrial and Applied Mathematics, 2nd edition, 2014.

R.W. Bent and P. Van Hentenryck. Waiting and relocation strategies in online stochastic vehicle routing. In IJCAI, pages 1816-1821, 2007.

R.W. Bent and P. Van Hentenryck. Scenario-based planning for partially dynamic vehicle routing with stochastic customers. Operations Research, 52:977-987, 2004.

E. Berhan, B. Beshah, D. Kitaw, and A. Abraham. Stochastic vehicle routing problem: A literature survey. Journal of Information $\&$ Knowledge Management, 13:1450022, 2014.

R. Moretti Branchini, V. Amaral Armentano, and A. Løkketangen. Adaptive granular local search heuristic for a dynamic vehicle routing problem. Computers \& Operations Research, 36:2955-2968, 2009.

J. Branke, M. Middendorf, G. Noeth, and M. Dessouky. Waiting strategies for dynamic vehicle routing. Transportation Science, 39:298-312, 2005. 
M. Christiansen, K. Fagerholt, B. Nygreen, and D. Ronen. Ship routing and scheduling in the new millennium. European Journal of Operational Research, 228:467-483, 2013.

F. Ferrucci, S. Bock, and M. Gendreau. A pro-active real-time control approach for dynamic vehicle routing problems dealing with the delivery of urgent goods. European Journal of Operational Research, 225:130-141, 2013.

G. Ghiani, G. Laporte, E. Manni, and R. Musmanno. Waiting strategies for the dynamic and stochastic traveling salesman problem. International Journal of Operations Research, 5:233-241, 2008.

G. Ghiani, E. Manni, A. Quaranta, and C. Triki. Anticipatory algorithms for same-day courier dispatching. Transportation Research Part E: Logistics and Transportation Review, 45:96-106, 2009.

A. Hemmati, L.M. Hvattum, I. Norstad, and K. Fagerholt. Benchmark suite for a class of industrial and tramp ship routing and scheduling problems. INFOR, 52: $28-38,2014$.

L.M. Hvattum, A. Løkketangen, and G. Laporte. Solving a dynamic and stochastic vehicle routing problem with a sample scenario hedging heuristic. Transportation Science, 40:421-438, 2006.

L.M. Hvattum, A. Løkketangen, and G. Laporte. A branch-and-regret heuristic for stochastic and dynamic vehicle routing problems. Networks, 49:330-340, 2007.

S. Ichoua, M. Gendreau, and J.-Y. Potvin. Exploiting knowledge about future demands for real-time vehicle dispatching. Transportation Science, 40:211-225, 2006.

J.E. Korsvik, K. Fagerholt, and G. Laporte. A tabu search heuristic for ship routing and scheduling. Journal of the Operational Research Society, 61:594-603, 2010.

S. Mitrović-Minić and G. Laporte. Waiting strategies for the dynamic pickup and delivery problem with time windows. Transportation Research Part B: Methodological, 38:635-655, 2004.

V. Pillac, M. Gendreau, C. Guéret, and A.L. Medaglia. A review of dynamic vehicle routing problems. European Journal of Operational Research, 225:1-11, 2013.

H.N. Psaraftis. Dynamic vehicle routing: Status and prospects. Annals of Operations Research, 61:143-164, 1995.

H.N. Psaraftis, M. Wen, and C.A. Kontovas. Dynamic vehicle routing problems: Three decades and counting. Networks, 2015.

V. Pureza and G. Laporte. Waiting and buffering strategies for the dynamic pickup and delivery problem with time windows. Information Systems and Operational Research, 46:165-176, 2008. 
U. Ritzinger, J. Puchinger, and R.F. Hartl. A survey on dynamic and stochastic vehicle routing problems. International Journal of Production Research, (ahead-ofprint):1-17, 2015.

M. Saint-Guillain, Y. Deville, and C. Solnon. A multistage stochastic programming approach to the dynamic and stochastic VRPTW. In Integration of AI and OR Techniques in Constraint Programming, pages 357-374. Springer, 2015.

J. Silberholz and B. Golden. Comparison of metaheuristics. In M. Gendreau and J.Y. Potvin, editors, Handbook of Metaheuristics, volume 146 of International Series in Operations Research and Management Science, pages 625-640. Springer, New York, 2nd edition, 2010.

B.W. Thomas. Waiting strategies for anticipating service requests from known customer locations. Transportation Science, 41:319-331, 2007.

G. Tirado, L.M. Hvattum, K. Fagerholt, and J.-F. Cordeau. Heuristics for dynamic and stochastic routing in industrial shipping. Computers and Operations Research, 40:253-263, 2013.

S. Vonolfen and M. Affenzeller. Distribution of waiting time for dynamic pickup and delivery problems. Annals of Operations Research, pages 1-24, 2014. 